\title{
Cystic transformation of cannon ball metastases in response to EGFR TKI
}

\author{
Satyajeet Sahoo, Prasanta Raghab Mohapatra, Saroj Kumar Das Majumdar, Mantyu Chhatria
}

All India Institute of Medical Sciences, Bhubaneswar, India

A 39-year-old never smoker male with a 3-month history of progressive dyspnea was found to have a heterogeneously enhancing right lung mass with multiple, well circumscribed rounded lesions of varying sizes, ranging from nodules to masses in both lungs, suggestive of cannon ball lesions [Figure 1 A-C]. Bronchoscopic biopsy revealed adenocarcinoma of the lung diffusely positive to thyroid transcription factor 1 (TTF1) and napsin. Contrast-enhanced computed tomography (CECT) scan of the abdomen and pelvis ruled out the presence of any synchronous tumor elsewhere. Epidermal growth factor receptor (EGFR) mutation analysis showed the presence of exon 19 deletion within the tumor specimen. The patient was staged as advanced disease and was hence started with $250 \mathrm{mg}$ of Gefitinib, an EGFR tyrosine kinase inhibitor (TKI) daily. Four months later CT scan showed shrunken primary lesion with multiple cysts of variable sizes bilaterally (Figure 1 D-F). Choriocarcinoma and renal cell carcinoma are the usual culprit of cannon ball metastases in the lungs. Other rare causes include prostate cancer, endometrial malignancy, synovial sarcoma, hepato-
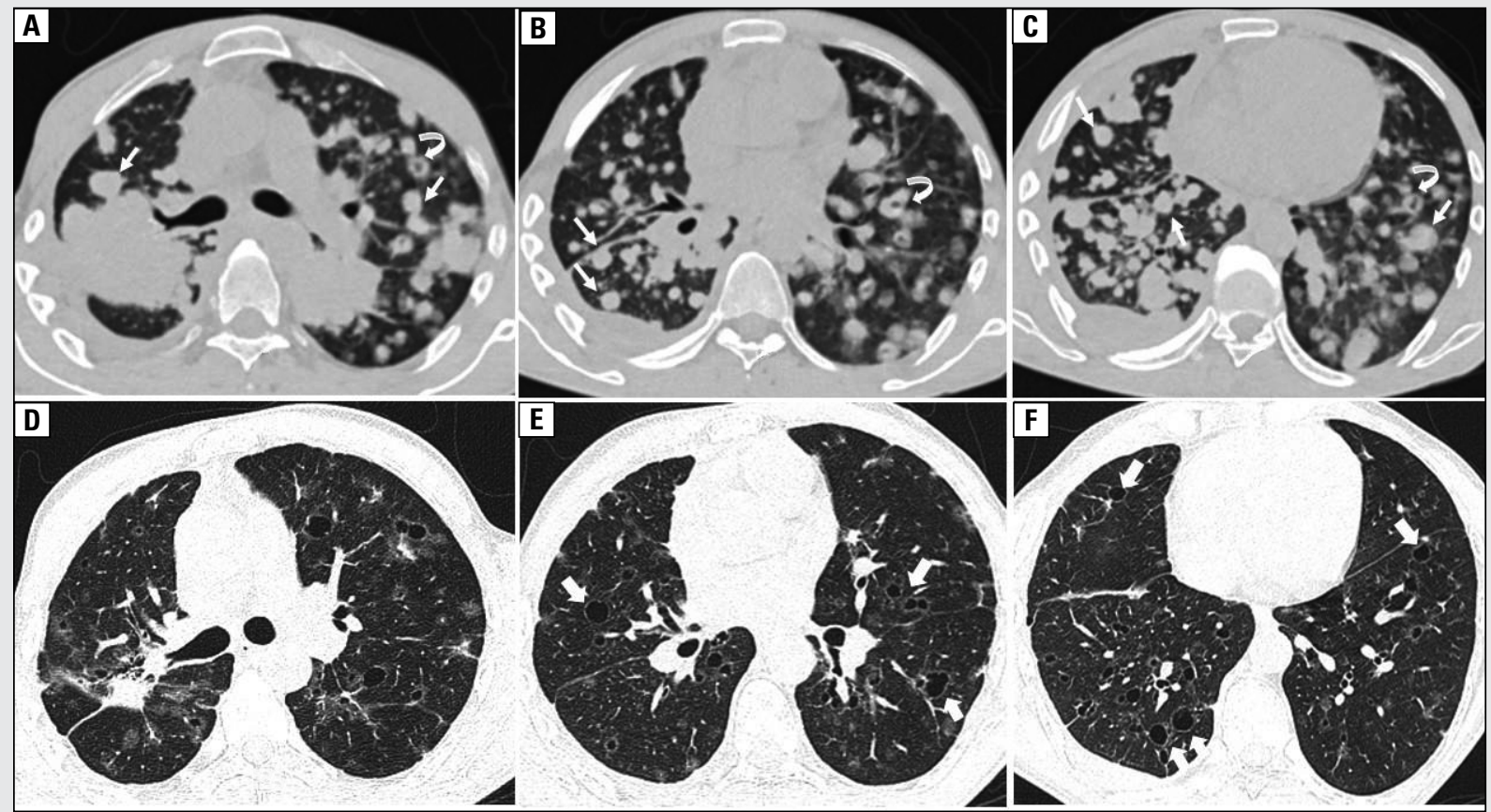

Figure 1. A-C: Axial CECT images (lung window) at different levels done before initiating EGFR TKI treatment showing heterogeneously enhancing lesion measuring $52 \times 51 \mathrm{~mm}$ in posterior segment of the right upper lobe (RUL) reaching up to the hilum, abruptly cutting off the RUL posterior segmental bronchus along with multiple nodular lesions consistent with cannon ball metastases (arrows) diffusely scattered without any lobar predilection. Few of these metastatic lesions have central cavitation (curved arrows). A small amount of pleural effusion is also seen; D-F: Axial CECT images at corresponding levels obtained after 4 months of EGFR TKI therapy showing reduced RUL mass lesion $(25 \times 12 \mathrm{~mm})$. Although the majority of cannon ball lesions have disappeared completely, some have transformed into thin-walled cysts of varying sizes (block arrows). Few areas are left with only ground glass nodules

Address for correspondence: Prasanta Raghab Mohapatra, Department of Pulmonary Medicine, AllMS, Bhubaneswar, Odisha, India 751019; e-mail: prmohapatra@hotmail.com

DOI: 10.5603/ARM.2019.0032

Received: 25.04 .2019

Copyright (C) 2019 PTChP

ISSN 2451-4934

Conflict of interest: none declared 
cellular and gastric carcinoma [1]. Multiple spherical lesions resembling cannon ball metastases can also be seen in sarcoidosis, tuberculosis, hydatid and fungal disease. Cannon ball metastases from a lung primary has been rarely reported. The behavior of these lesions with treatment was noteworthy in this case. A cystic transformation of the metastatic deposits was noticed in follow-up scans. Cystic lung changes secondary to EGFR TKI treatment have been a rare description. This cystic transformation of metastatic deposits may be a class effect of EGFR TKIs in a selected subset of patients. It has been hypothesized that the transformation can be due to anti-angiogenic effects of these TKIs or tumor lysis by TKIs [2]. Human alveolar epithelial type 2 pneumocytes, which have an active role in repairing of alveolar walls, have a high EGFR expression. Hindrance of this pathway by EGFR TKI leads to a poor or lack of parenchymal generation resulting in cystic changes. These cystic changes can also be evident as a part of EGFR TKI- induced interstitial lung disease (ILD), in which case the patient develops or has progressive respiratory symptoms. However, in our case, we do not presume it to be an ILD as our patient had a remarkable improvement in symptoms. This case emphasizes that the differentials of cannon ball pulmonary metastases should include lung as one of the differentials of primaries and how these metastatic lesions can transform to cysts with EGFR TKIs, which can be difficult to distinguish radiologically from drug-induced ILD.

\section{References:}

1. Ammannagari N, Polu V. ‘Cannon ball’ pulmonary metastases. BMJ Case Rep. 2013; 2013, doi: 10.1136/bcr-2012-008158, indexed in Pubmed: 23302554

2. Ansari J, Batubara E, Ali M, et al. Atypical diffuse bilateral cystic lung changes secondary to erlotinib treatment in a patient with metastatic non-small cell lung carcinoma: A case report and literature review. Mol Clin Oncol. 2018; 9(1): 92-95, doi: 10.3892/ mco.2018.1620, indexed in Pubmed: 29977544. 